\title{
Cicloergometria adaptada para membros superiores de pacientes hemiparéticos por acidente vascular cerebral: uma série de casos
}

\author{
Adapted cycle ergometry for upper limbs of hemiparetic \\ stroke patients: a case series
}
Cicloergometria adaptada para miembros superiores de pacientes hemiparéticos debido a accidente cerebrovascular: una serie de casos

\author{
Antonio Vinicius Soares ${ }^{1}$, Rafaela Korn ${ }^{2}$, Tássia Pertile ${ }^{3}$, Bruna de \\ Domenico ${ }^{4}$, Fernando Luis Fischer Eichinger ${ }^{5}$, Fabrício Noveletto ${ }^{6}$
}

\begin{abstract}
1.Fisioterapeuta, Doutor em Ciências do Movimento Humano. Professor da Universidade da Região de Joinville e da Faculdade Ielusc, Joinville-SC, Brasil. https://orcid.org/0000-0001-6090-1423

2.Fisioterapeuta pela Faculdade Guilherme Guimbala, FGG, Joinville-SC, Brasil. https://orcid.org/00000002-0243-5134

3.Fisioterapeuta pela Faculdade Guilherme Guimbala, FGG, Joinville-SC, Brasil. https://orcid.org/00000001-7614-7840

4.Fisioterapeuta pela Faculdade Guilherme Guimbala, FGG, Joinville-SC, Brasil. https://orcid.org/0000$\underline{0002-4271-1236}$

5.Fisioterapeuta, Mestre em Ciências do Movimento Humano, Professor da Faculdade Guilherme Guimbala e da Universidade da Região de Joinville, UNIVILLE, Joinville-SC, Brasil. https://orcid.org/0000-0002$4385-4853$

6.Engenheiro Eletricista, Doutor em Engenharia Elétrica, Professor da Faculdade Guilherme Guimbala e da Universidade do Estado de Santa Catarina - UDESC, Joinville-SC, Brasil. http://orcid.org/0000-0003-03104262
\end{abstract}

\section{Resumo}

Introdução. O Acidente Vascular Cerebral (AVC) é uma das principais causas de morte e incapacidade. Método. Uma série de casos com quatro participantes. Os testes realizados foram Dinamometria dos músculos flexores de cotovelo, força de preensão manual, Manovacuometria (Pressão expiratória máxima), Escala de Ashworth Modificada e Perfil de Saúde de Notthinghan. O programa ocorreu 2 vezes por semana durante 8 semanas (16 sessões). Resultados. Foram observados ganhos na maioria dos parâmetros avaliados. Conclusão. Esta técnica é de fácil utilização e baixo custo, sendo promissora para a recuperação do membro superior de pacientes hemiparéticos por AVC.

Unitermos. Acidente Vascular Cerebral; Hemiparesia; Cicloergometria

\begin{abstract}
Introduction. Stroke is a major cause of death and disability. Method. A series of cases with four participants. The tests carried out were dynamometry of the elbow flexor muscles, handgrip strength (HGS), Manovacuometry (Maximum expiratory pressure), Modified Ashworth Scale and Notthinghan Health Profile. The program took place twice a week for 8 weeks (16 sessions). Results. Gains were observed in most of the evaluated parameters. Conclusion. This technique is easy to use and low cost, promising for the recovery of the upper limb of hemiparetic stroke patients.
\end{abstract}

Keywords. Stroke; Hemiparesis; Cyclo ergometry 


\section{Resumen}

Introducción. El accidente cerebrovascular es una causa importante de muerte y discapacidad. Método. Una serie de casos con cuatro participantes. Las pruebas realizadas fueron dinamometría de los músculos flexores del codo, fuerza de agarre, manovacuometría (presión espiratoria máxima), escala de Ashworth modificada y perfil de salud de Notthinghan. El programa tuvo lugar dos veces por semana durante 8 semanas (16 sesiones). Resultados. se observaron ganancias en la mayoría de los parámetros evaluados. Conclusión. Esta técnica es fácil de usar y de bajo costo, prometedora para la recuperación de la extremidad superior de los pacientes hemiparéticos debido a un accidente cerebrovascular.

Palabras clave: accidente cerebrovascular; Hemiparesia; Cicloergometría

Trabalho realizado na Faculdade Guilherme Guimbala, Joinville-SC, Brasil.

\section{INTRODUÇÃO}

O AVC é considerado um evento vascular agudo que ocasiona lesão no tecido cerebral, representando uma das principais causas de morte e incapacidade ${ }^{1,2}$. A cada ano, no mundo, cerca de 15 milhões de pessoas sofrem um $\mathrm{AVC}^{3}$.

A incapacidade ocasionada pelo AVC gera disfunções sensoriais, no equilíbrio, coordenação, comunicação, cognição, e, sobretudo diferentes graus de déficits motores ${ }^{4}$. A cicloergometria adaptada é uma forma comumente utilizada como treino aeróbio em pacientes que sofreram AVC, pois oferece maior estabilidade para o tronco, sendo assim, uma forma mais segura de treinamento ${ }^{5}$. Através da cicloergometria podem-se obter benefícios, como redução da fadiga, aumento da resistência aeróbia e incremento da força muscular. Desta forma, esta técnica pode facilmente ser adicionada ao programa de tratamento destes pacientes. 
Os efeitos terapêuticos da cicloergometria sobre os MMSS pode representar uma nova estratégia de tratamento para esses pacientes, uma vez que o comprometimento do MMSS é sempre um desafio para a equipe de reabilitação.

Com base nesta argumentação, o objetivo deste estudo foi avaliar os efeitos terapêuticos da cicloergometria adaptada para os MMSS em pacientes hemiparéticos por AVC.

\section{MÉTODO}

\section{Relato dos casos}

O presente estudo relata uma série de casos, com três avaliações de pré-teste, dezesseis sessões de treinamento com o cicloergômetro adaptado, e três reavaliações de póstestes. São relatados quatro casos, todos de homens hemiparéticos por AVC, destes, todos destros e hemiparéticos a direita. A idade média foi de $54 \pm 7$ anos, e tempo de lesão de $22,8 \pm 15,4$ meses. Todos eram atendidos no Núcleo de pesquisa em Neurorreabilitação (NUPEN) localizada na Faculdade Guilherme Guimbala, na cidade de Joinville-SC.

O estudo foi aprovado sob o número 68560017.6.0000.5365 pelo Comitê de Ética em Pesquisa em Seres Humanos da Universidade da Região de Joinville. Todos os participantes assinaram um Termo de Consentimento Livre e Esclarecido. 


\section{Procedimentos}

- Mini Exame do Estado Mental: Para avaliar a função cognitiva e rastreamento de quadros demenciais. O ponto de corte é de 23/24, tem mostrado alta capacidade de discriminação de indivíduos cognitivamente alterados ${ }^{6}$.

- Escala de Ashworth Modificada (EAM): Foi utilizado para avaliação dos músculos espásticos ${ }^{7}$. Gradua a espasticidade de 0 a 5 pontos, sendo o grau 0 hipotônico e o grau 5 rigidez do segmento corporal7, no presente estudo foi avaliado o tônus muscular dos flexores do cotovelo (músculos bíceps braquial, braquial e braquiorradial).

- Manovacuometria: Para avaliar a força muscular inspiratória e expiratória máxima, realizada através da estática de boca (PImáx e PEmáx) ${ }^{8-10}$. No presente estudo, utilizou-se a manovacuometria para avaliar apenas a PEmáx. - Perfil de Saúde de Nothinghann (PSN): Trata-se de um questionário auto administrado, constituído de 38 itens, com respostas no formato sim/não, englobando seis categorias como, nível de energia, dor, reações emocionais, sono, interação social e habilidades físicas ${ }^{11}$.

- Dinamometria: A avaliação da FPM foi com o dinamômetro Takei, a mensuração foi conforme recomendações da Associação Americana de Terapeutas da Mão ${ }^{12}$ e a mensuração do músculo flexores do cotovelo foi utilizado o dinamômetro Chatillon ${ }^{13}$. Após a realização de duas medidas de aproximadamente 5 segundos em cada grupo muscular, a melhor medida é registrada. 
Para monitorização das sessões de treinamento foram usados o Oxímetro de pulso Geratherm que é um método não invasivo de medir a saturação de oxigênio ${ }^{14}$ e a Escala de Esforço Percebido Modificada de Borg para monitorar a intensidade do exercício durante a fase de treinamento, sendo que acima de 7 é indicativo de encerramento do exercício.

Para o treinamento foi utilizado uma adaptação do Cicloergômetro Horizontal Atthletic Way ${ }^{15}$, onde no lugar dos pedais foram adaptados com goteiras para as mãos, foi fixado sobre uma mesa na altura dos ombros. Detalhes das adaptações são apresentados na Figura 1.

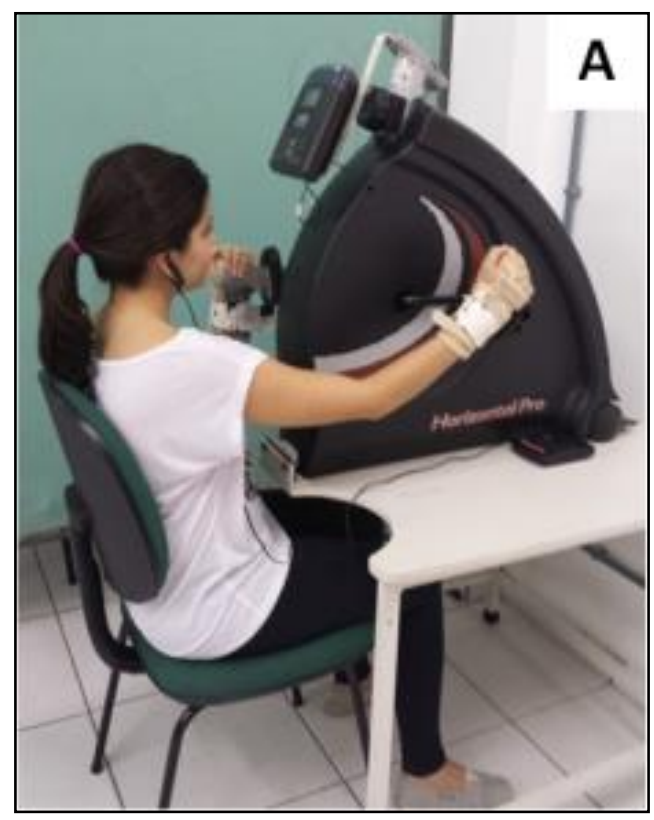

Figura 1. Cicloergômetro adaptado para os membros superiores.

O treinamento foi determinado segundo as orientações de Soares ${ }^{15}$, através das fases de aquecimento com duração de 5 minutos e potência de $25 \mathrm{~W}$, fase de resistência de 20 a $60 \mathrm{~min}$, com incremento de $25 \mathrm{~W}$ a cada 5 minuto, 
desaquecimento de 5 minutos em $25 \mathrm{~W}$, e a velocidade constante de $40 \mathrm{rpm}$ ( $\approx 8$ a $8,5 \mathrm{~km} / \mathrm{h}$ ). Sendo assim, foi determinado a FC Máxima (220-idade) e zona alvo de treinamento entre $70-90 \%$ da FC Máxima.

\section{Análise dos dados}

A tabulação e análise dos dados foi realizada no software GraphPad Prism $6^{\circledR}$. Foram obtidos dados da estatística descritiva como as médias e desvios padrão. 0 índice de desempenho foi calculado com base na diferença percentual entre as medidas de pré e pós-testes.

\section{RESULTADOS}

Dos quatro participantes da pesquisa, três eram hemiparéticos à direita, e um à esquerda. Todos estavam dentro dos critérios de inclusão do estudo. Nenhum deles apresentou traços demenciais detectados pelo Mini Exame do Estado Mental.

A Tabela 1 apresenta a análise estatística dos resultados dos pacientes envolvidos no estudo. São mostrados dados da estatística descritiva como média e desvio padrão, e os índices de desempenho traduzidos pela diferença percentual entre os pré e pós-testes de cada variável controlada. 
Tabela 1. Resultados dos pacientes envolvidos na pesquisa.

\begin{tabular}{lccc}
\hline Variáveis & Pré & Pós & ID (\%) \\
\hline EAM (FC) & 2,3 & 1,5 & 33,3 \\
DIN (FC dir.) & 9,3 & 15,8 & 69,9 \\
DIN (FC esq.) & 11,6 & 18,4 & 58,6 \\
FPM dir. & 18,7 & 22,5 & 20,3 \\
FPM esq. & 28,9 & 33,2 & 14,9 \\
PSN & 7,3 & 6,5 & 11,0 \\
MV (PEmax) & 83,8 & 88,8 & 6
\end{tabular}

EAM: Escala de Ashworth Modificada; FC: Flexores de cotovelo (0-5); DIN: Dinamometria- flexores de cotovelo; FPM: Dinamometria da força de preensão manual (kgf); PSN: Perfil de Saúde de Nottinghan (0-38); MV- PEmax: Manuvacuometria - Pressão expiratória máxima $(\mathrm{mmHg})$; ID: Índice de desempenho (diferença percentual entre pré e pós-testes).

Na Figura 2 são comparadas as médias de tempo inicial (3 primeiras sessões) e a média final (3 últimas sessões) de cada um dos 4 participantes do estudo.

Pode-se observar que houve um incremento de tempo e carga de treinamento. No início a média de tempo era de 13,4 minutos (25W), alcançando no final 17,1 minutos (50 W), representando um aumento de $27,6 \%$.

Figura 2. Tempo de treinamento no cicloergômetro. 


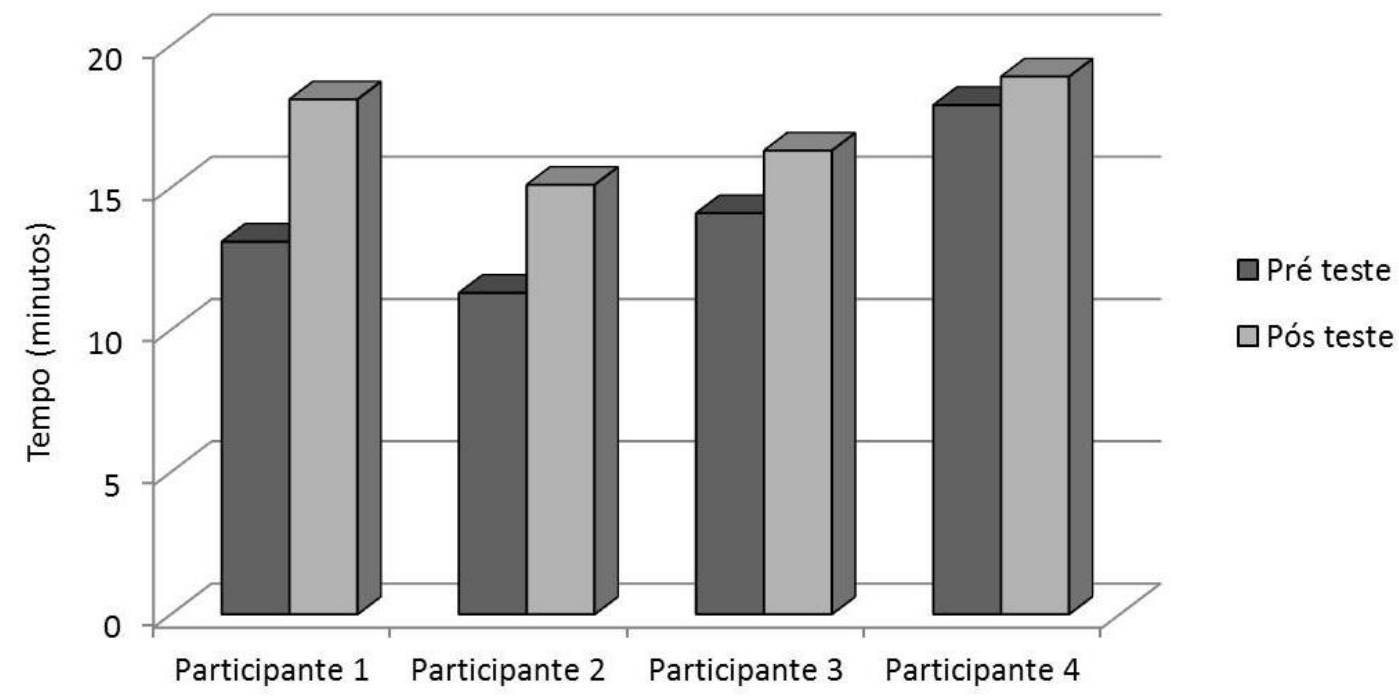

\section{DISCUSSÃO}

A cicloergometria é uma técnica empregada no ambiente clínico e em pesquisas, mas a sua maior aplicabilidade é na reabilitação dos membros inferiores ${ }^{15}$. Na sequência serão discutidos os resultados em relação a cada variável controlada.

Quanto à espasticidade, houve uma redução discreta não significativa nos músculos flexores do cotovelo. Algo já observado em estudo anterior ${ }^{15}$.

Em relação às medidas de força muscular dos flexores do cotovelo foi observado um incremento. Isto é relevante, pois a deficiência da força muscular pode causar impacto significativo na execução das atividades de vida diária, sendo que a fraqueza nos MMSS tem impacto na independência funcional do paciente ${ }^{16}$. Em outro estudo que utilizou 0 cicloergômetro de MMSS, apresentou como resultados 0 aumento da força muscular e da amplitude dos movimentos 
do ombro e cotovelo no hemicorpo afetado, tendo relação com a independência funcional destes pacientes ${ }^{17}$.

Os exercícios de fortalecimento muscular atuam sobre o recrutamento de unidades motoras, melhorando a capacidade e o tempo de geração da força, reduzindo a rigidez muscular e a hiperatividade reflexa, mantendo a extensibilidade funcional dos músculos ${ }^{18}$.

Embora alguns estudos usando a técnica para os membros inferiores em hemiparéticos tenham encontrado incrementos significativos na manovacuometria, e resultando em importantes repercussões no condicionamento cardiorrespiratório ${ }^{15}$. Nessa série de casos as medidas de PeMax aumentaram discretamente.

A percepção da qualidade de vida não apresentou alteração significativa pelo PSN. Tal escala apresenta alto índice de confiabilidade, porém se refere a um instrumento genérico, menosprezando o impacto na condição de saúde, como o AVC, por serem menos sensíveis sobre a incapacidade da doença ${ }^{19}$.

Como em outros estudos, porém, usando a cicloergometria para os membros inferiores em hemiparéticos, importante melhora no condicionamento aeróbio com aumento no tempo e na carga no treinamento também foram encontrados, no que resultou em aumento da capacidade aeróbia, da tolerância ao exercício e a diminuição dos níveis de pressão arterial ${ }^{15}$. 


\section{CONCLUSÃO}

A análise dos resultados obtidos com o treinamento do cicloergômetro adaptado para MMSS resultou melhoras significativas nas variáveis controladas nesta série de caso.

Esta técnica é de fácil utilização e baixo custo, sendo promissora para a recuperação do membro superior de pacientes hemiparéticos por AVC. Pode ser associada aos exercícios convencionais para incrementar o condicionamento aeróbio, a recuperação da força muscular, e assim, aumentar a independência funcional destes pacientes.

\section{REFERÊNCIAS}

1.Pan B, Jin X, Jun L, Qiu S, Zheng Q, Pan M. The relationship between smoking and stroke: a meta-analysis. Medicine 2019;98:e14872. http://dx.doi.org/10.1097/MD.0000000000014872

2.Song K, Wang L, Wu W. Mental Practice for upper limb motor restoration after stroke: an update meta-analysis of randomized controlled trials. Top Stroke Rehab 2019;26:87-93. http://dx.doi.org/10.1080/10749357.2018.1550613

3.Thrift AG, Cadilhac DA, Thayabaranathan T, Howard G, Howard VJ, Rothwell PM, et al. Global Stroke Statistics. Inter J Stroke 2014;9:618. http://dx.doi.org/10.1111/ijs. 12245

4.Lima AGT, Petribú K. Acidente vascular encefálico: revisão sistemática sobre qualidade de vida e sobrecarga de cuidadores. Rev Bras Neurol Psiq 2016;20:253-66.

https://www.revneuropsiq.com.br/rbnp/article/view/168/107

5.Sibley KM, Tang A, Brooks D, Brown DA, Mcllroy WE. Feasibility of adapted aerobic cycle ergometry tasks to encourage paretic limb use after stroke: a case series. J Neurol Phys Ther 2008;32:80-7. http://dx.doi.org/10.1097/NPT.0b013e318176b466

6. Lourenço RA, Veras RP. Mini-Exame do Estado Mental: características psicométricas em idosos ambulatoriais. Rev Saúde Pub 2006;40:7129. http://dx.doi.org/10.1590/S0034-89102006000500023

7.Bakheit AM. Optimising the methods of evaluation of the effectiveness of botulinum toxin treatment of post-stroke muscle spasticity. J Neurol Neuros Psych 2004;75:665-6. http://dx.doi.org/10.1136/jnnp.2003.026096 
8. Maria NNS, Zanelli EM, Silva MB, Asa SKP, Fávero FM, Fukujima, MM, et al. Tests used to evaluate respiratory function in neuromusculares diseases. Rev Neurocienc 2007;15:60-9. http://dx.doi.org/10.34024/rnc.2007.v15.8725

9. Perrin C, Unterborn JN, Ambrósio C, Hill NS. Pulmonary complications of chronic neuromuscular disease and their management. Muscle Nerve 2004;29:5-27. http://dx.doi.org/10.1002/mus.10487

10.Bruschi C, Cervai I, Zoia MC, Fanfulla F, Fiorentini M, Cosali L. Reference values of maximal inspiratory mouth pressure: a population based study. Am Rev Respirat Dis 1992;146:790-3. http://dx.doi.org/10.1164/ajrccm/146.3.790

11.Teixeira-Salmela LF, Magalhães LC, Souza AC. Adaptação do Perfil de Saúde de Nottingham: um instrumento simples de avaliação de qualidade de vida. Cad Saúde Púb 2004;20:905-14. http://dx.doi.org/10.1590/S0102-311X2004000400004

12.Soares AV, Carvalho JM, Fachini J, Domenech SC, Borges JNG. Correlação entre os testes de dinamometria de preensão manual, escapular e lombar. Acta Bras Mov Hum 2012;2:65-72. http://www.periodicos.ulbra.br/index.php/actabrasileira/article/view/ $2956 / 2218$

13.Andrews AW, Thomas MW, Bohannon RW. Normative values for isometric muscle force measurements abtained with hand-held dynamometers. Phys Ther 1996;76:248-59. http://dx.doi.org/10.1093/ptj/76.3.248

14.Parsons PE, Heffner JE. Segredos em pneumologia: respostas necessárias ao dia-a-dia em rounds, na clínica, em exames orais e escritos. ( $2^{\circ}$ ed.). Porto Alegre: Artmed, 2000.

15.Soares AV, Gesser E, Andrade C, Mesadri TJ, Santos SBO, Júnior NGB. Cicloergometria Adaptada para Pacientes Hemiparéticos por Acidente Vascular Cerebral. Arq Cat Med 2016;45:108-16. http://www.acm.org.br/acm/seer/index.php/arquivos/article/view/66 $\angle 124$

16. Franciulli PM, Magaldi CM, Bigongiari A, Barbanera M. Efeito do treinamento resistido em hemiparéticos crônicos no equilíbrio e torque isocinético do joelho. Rev Bras Ciênc Saúde 2018;22:125-30. http://dx.doi.org/10.22478/ufpb.2317-6032.2018v22n2.32752

17.Diserens K, Perret N, Chatelain S, Bashir S, Ruegg D, Vuadens P, et al. The effect of repetitive arm cycling on post stroke spasticity and motor control: repetitive arm cycling and spasticity. J Neurol Sci 2007;253:18-24. http://dx.doi.org/10.1016/j.jns.2006.10.021

18. Barbosa DD, Thojahn MT, Porto DVG, Hentschke GS, Hentschke VS. Strength training protocols in hemiparetic individuals post stroke: a systematic review. Fisioter Mov 2018;31. http://dx.doi.org/10.1590/1980-5918.031.ao27

19.Cabral DL, Damascena CG, Teixeira-Salmela LF, Laurentino GEC. Confiabilidade do Perfil de Saúde de Nottingham após acidente vascular encefálico. Ciênc Saúde Col 2012;17:1313-22. http://dx.doi.org/10.1590/S1413-81232012000500025 
